\section{A propósito de un esguince de mala evolución}

\section{Sr. Director:}

Hemos leído con gran interés el artículo publicado en su revista, y nos parece oportuno realizar una serie de consideraciones, con el ánimo de ayudar a conseguir conceptos claros en lo referente a anamnesis, exploración, y sobre todo diagnóstico diferencial ante el esguince de tobillo.

Los autores sugieren que "ante un esguince que no evoluciona satisfactoriamente debemos pensar en descartar otras patologías no detectadas en un primer momento". En realidad, casi la mitad de los esguinces no evolucionan bien, aproximadamente el $40 \%$ de los pacientes presentan algún tipo de secuelas (dolor residual, inestabilidad mecánica o funcional) hasta un año después del episodio ${ }^{1,2}$. El hecho de que persista el dolor más allá de 4-6 semanas, como dicen los autores, entraría dentro de ese $40 \%$ de esguinces con secuelas, y no por ello obligaría a reinterrogar al paciente ni a reexplorarle. Debemos realizar una buena historia y una exploración completa desde el primer momento. Y cuando decimos completa nos referimos a descartar la posible existencia de complicaciones que pueden surgir asociadas de algún modo al traumatismo que produjo el esguince. Como por ejemplo fracturas osteocondrales, rotura del retináculo de los peroneos, fractura de la base del $5^{\circ}$ meta, fractura del "os trigonum", "impin gement" sinovial, $\mathrm{y}$, por supuesto, rotura parcial o completa del tendón de Aquiles.

En lo referente a los hallazgos exploratorios, el hecho de que exista una imposibilidad para mantenerse correctamente "de puntillas", puede sugerir afectación del tendón de Aquiles, pero no debemos olvidar, que en un esguince grave de tobillo (III o incluso en ocasiones
II) puede aparecer este mismo hallazgo exploratorio. De hecho, en los esguinces, si el enfermo es capaz de mantenerse "de puntillas" o en apoyo monopodal (signo de Freeman) sobre el tobillo lesionado, supone un signo de buen pronóstico, ya que implica que la propiocepción no se ha visto especialmente afectada.

En la anamnesis deben recogerse, efectivamente, todos los datos que proponen los autores, y algunos más que pueden sernos de utilidad; la existencia de esguinces previos supone el doble de posibilidades de padecer un esguince en el futuro, la aparición de dolor intenso, brusco y breve, debe hacernos sospechar la rotura completa del ligamento, ya que se rompen también las terminaciones sensitivas, e inmediatamente desaparece el dolor, permaneciendo entonces tan sólo la inestabilidad y el enorme edema. El aumento de más de 4 $\mathrm{cm}$ en el perímetro del tobillo lesionado con respecto al tobillo sano, supone la rotura ligamentosa hasta en el $70 \%$ de las oca- 
siones, según algunos autores. Se deben explorar sistemáticamente, independientemente de cual sea el diagnóstico de sospecha, buscando dolor, crepitación o inestabilidad las siguientes estructuras: hueso navicular, cola del $5^{\circ}$ meta, la zona central en lugar del borde posterior de los últimos $6 \mathrm{~cm}$ de ambos maleolos (esta maniobra de desplazar la zona a explorar incrementa la especificidad para la petición de Rx y descartar la existencia de fracturas) ${ }^{3}$, el tendón aquíleo, los tendones de los peroneos, el tendón del tibial anterior, la sindésmosis TPA, y las articulaciones mediotarsianas (Chopart y Lisfranc), y por supuesto se debe explorar el tobillo "desde atrás", desde la región posterior. Recordemos que el LLE es una estructura extraarticular, y si sólo existe esta lesión, los espacios retroaquíleos se hallarán libres; no siendo así en el caso de afectación aquílea o de estructuras intraarticulares ${ }^{4}$.

Con respecto al tratamiento que los autores proponen, cierto es que en los grados I y II debe ser lo más funcional posible. Parece más eficaz el uso desde los primeros momentos de vendajes funcionales, evitando férulas rígidas ${ }^{5-7}$, lo que supondría una mucho más rápida recuperación y una mucho mejor cicatrización de la lesión; excepto si existe dolor severo o un gran edema. En cuanto a la rotura total, efectivamente, no existe acuerdo. Se realiza cirugía electiva en atletas de competición. En el resto, siempre es preferible realizar una buena rehabilitación durante 6-8 semanas tras la retirada del yeso y reevaluar la procedecencia o no de la reparación quirúrgica ${ }^{8}$.

Para concluir, nos parece oportuno felicitar a los autores por el caso presentado, que se nos antoja francamente interesante e ilustrativo para aquéllos de nosotros a los que nos ilusiona la "traumatología menor".

\section{BIBLIOGRAFÍA}

1. Salcedo I, Sánchez A, Carretero B, Herrero M, Mascías C, Panadero Carlavilla FJ. Esguince de tobillo. Valoración en Atención Primaria. Med. Integr 2000; 36: 45-52.

2. Nielsen $A B, Y$ de J. Epidemiology and traumatology of injuries in soccer. Am J Sports Med 1980: 8; 235

3. Leddy JJ, Smolinsky RJ, Lawrence J, Snyder JL, Priore RL. Prospective evaluation of the Ottawa ankle rules in a University Sports Medicine Center. With a modification to increase specificity for identifying malleolar fractures Am J Sports Med 1998; 26 (2): 158-65.

4. Dunowski R, Chanussot JC. Manual de traumatología del deporte. París: Masson 1992; 233-9.

5. Gómez-Castresana F, Glez del Pino J, eds. Actualización en traumatología y cirugía ortopédica 3. Orthopaedic Knowledge Update (AAOS) SECOT Madrid: Garsi, 1992; 59-68.

6. De Felipe Gallego J, Salvat Salvat I, Mayoral del Moral O, Rez Sanchez JC Tratamiento funcional de los esguinces de tobillo grado I-II. Madrid: Mapfre Medicina, 1995; 6: 165-72.

7. Hazañas Ruiz S, Gálvez Alcaraz L, Cepas Soler JA. Estabilización funcional frente a inmovilización ortopédica en el esguince de tobillo grado I-II (leve). At. Prim 1999; 23: 425-8.

8. Brostrom L. Sprained ankles. V . Treatment and prognosis in recent ligament ruptures 1996; 132 (5): 537-50.

M. Esteve Sacristán, J. Ca ballero Jiménez, G. Gómez-Zorita, O. Morán Homa*, F. J. P anadero**

Residentes de MFyC. *Médico de Familia. Tutor de Residentes. **Médico de Familia. Especialista en Medicina Deportiva. Tutor de Residentes. EAP A rgüelles. Madrid.

\section{Evaluación de las actividades de cirugía menor realizadas en el centro de salud}

\section{Sr. Director:}

La cirugía menor (CM) comprende procedimientos quirúrgicos sencillos y de corta duración, que se realizan en los tejidos superficiales y accesibles, bajo anestesia local y con complicaciones postquirúrgicas escasas y poco importantes a priori $^{1}$.

Existe un interés creciente por la CM desarrollada en el ámbito de Atención Primaria. La CM ha sido incluida en el programa de formación de residentes de Medicina de Familia y en la Cartera de Servicios del Insalud desde 1999.

En nuestro centro de salud se practica CM desde 1994. En enero de 1998 se puso en marcha la consulta programada de cirugía menor. A continuación se exponen las actividades de CM realizadas en el Centro de Salud Periodistas I desde enero de 1998 hasta marzo de 2000.

El EAP Periodistas I atiende a una población urbana de 14.000 habitantes. En 1998 se establece una consulta programada de CM, en agenda 75 minutos/un día a la semana. Los pacientes eran captados en la consulta a demanda y citados para la consulta programada de CM.

Se diseñó un estudio descriptivo retrospectivo. Fueron revisadas las historias clínicas de los pacientes que habían sido sometidos a una intervención de CM entre enero de 1998 y marzo de 2000. Las variables cualitativas se analizaron mediante porcentajes simples y las variables cuantitativas mediante estadísticos descriptivos. Las variables evaluadas fueron edad, sexo, procedimiento quirúrgico empleado, diagnóstico anatomopatológico, concordancia clínico-patológica, tiempo de demora desde que el paciente consultaba hasta la cirugía y complicaciones.

El número de intervenciones realizadas fue 54, 34 mujeres $(62,9 \%)$ y 20 varones $(37,1 \%)$. La edad media de los pacientes fue 45 años (rango 15-81). En cuanto al procedimiento quirúrgico aplicado, se realizó escisión fusiforme en 17 casos $(31,4 \%)$, afeitado/curetaje en 22 casos $(40,74 \%)$, punch/biopsia 